PROCEEDINGS OF THE

AMERICAN MATHEMATICAL SOCIETY

Volume 103, Number 1, May 1988

\title{
WEAK $(1,1)$ BOUNDEDNESS OF SINGULAR INTEGRALS WITH NONSMOOTH KERNEL
}

\author{
STEVE HOFMANN
}

(Communicated by Richard R. Goldberg)

\begin{abstract}
If $\Omega \in L^{q}\left(S^{1}\right)$ for some $q>1, \int_{S^{1}} \Omega=0$, and $\Omega$ is homogeneous of degree 0 , then the operator defined in two dimensions by $T_{\varepsilon} f(x)=$ $\int_{\varepsilon>0 \text {. }} f(x-y) \Omega(y)|y|^{-2} d y$ is of weak-type $(1,1)$ with bound independent of
\end{abstract}

Consider the two-dimensional operator defined on $L^{P}, 1 \leq p<\infty$, by

$$
T_{\varepsilon} f(x) \equiv \int_{|y|>\varepsilon} f(x-y) \Omega(y)|y|^{-2} d y
$$

where $\Omega$ is homogeneous of degree zero, belongs to $L^{q}$ on the unit circle for some $q>1$, and has mean value zero. Note that no smoothness assumptions are placed upon $\Omega$. It is well known [C-Z] that $T_{\varepsilon}$ is of type $(p, p)$ with bound independent of $\varepsilon$ for $1<p<\infty$. The proof is by the method of rotations. It has been an open problem, however, to determine the behavior of $T_{\varepsilon}$ acting on $L^{1}$. The main result of this paper is the following:

THEOREM. Let $\Omega$ and $T_{\varepsilon}$ be defined as above. Then $T_{\varepsilon}$ is of weak-type $(1,1)$. More explicitly: $\left|\left\{\left|T_{\varepsilon} f\right|>\alpha\right\}\right| \leq c\|\Omega\|_{q} \alpha^{-1}\|f\|_{1}$ where $c$ is an absolute constant independent of $\varepsilon$.

The proof of this theorem is based on ideas developed by Michael Christ in [C]. He proves that the related operator defined by

$$
M_{\Omega} f(x) \equiv \sup _{r>0} \frac{1}{r^{2}} \int_{|y|<r}|f(x-y)||\Omega(y)| d y
$$

is weak $(1,1)$. What is new in this paper is the estimate for the cross terms (2), which do not arise in the analysis of $(*)$. The proof of (2) uses an important observation contained in $[\mathbf{C}]$; namely that a convolution of two kernels of the form $K=\Omega \psi$ (where $\psi \in C_{0}^{\infty}$ with annular support) can be expressed as the integral over $S^{1} \times S^{1}$ of the convolution of two singular measures (3).

I would like to acknowledge the joint work of M. Christ and J. L. Rubio de Francia, who have obtained independently the result contained herein. I would also like to thank the referee for valuable comments.

ProOF OF THEOREM. We may assume $\Omega \in L^{\infty}\left(S^{1}\right)$. The general case follows by a limiting argument. We may also assume $\|\Omega\|_{q}=1$. Fix $\alpha>0$ and $f \in L^{1}$. Perform a standard Calderon-Zygmund decomposition to obtain nonoverlapping

Received by the editors March 30, 1987.

1980 Mathematics Subject Classification (1985 Revision). Primary 42B20.

(C) 1988 American Mathematical Society $0002-9939 / 88 \$ 1.00+\$ .25$ per page 
dyadic cubes $\left\{Q_{n}\right\}$ and functions $g$ and $b$ so that $f=g+b,\|g\|_{2}^{2} \leq c \alpha\|f\|_{1}$, $b=\sum_{n} b_{n}, \operatorname{supp} b_{n} \subseteq Q_{n},\left\|b_{n}\right\|_{1} \leq c \alpha\left|Q_{n}\right|, b_{n}$ has mean value zero and $\left|\bigcup_{n} Q_{n}\right| \leq$ $\alpha^{-1}\|f\|_{1}$.

Let $E \equiv \bigcup_{n} 5 Q_{n}$, where $5 Q_{n}$ has the same center as $Q_{n}$ but side length 5 times that of $Q_{n}$. Then $|E| \leq C \alpha^{-1}\|f\|_{1}$.

As is well known, it is enough to show that

$$
\left|\left\{x \in E^{c}:\left|T_{\varepsilon} b(x)\right|>\alpha\right\}\right| \leq(c / \alpha)\|f\|_{1} .
$$

Define $B_{i} \equiv \sum b_{n}$, where the sum runs over those $n$ for which $\left|Q_{n}\right|=2^{2 i}$. Let $\varphi \in C_{0}^{\infty}\left(\frac{1}{4}, 1\right), \varphi \geq 0$ and $\sum_{j=-\infty}^{\infty} \varphi\left(x / 2^{j}\right) \equiv 1$, for all $x>0$. Let $K_{0}(x) \equiv$ $\left(\Omega(x) /|x|^{2}\right) \varphi(|x|)$, and let $K_{j}(x)=2^{-2 j} K_{0}\left(2^{-j} x\right)=\left(\Omega(x) /|x|^{2}\right) \varphi\left(2^{-j}|x|\right)$. Note that

$$
\left|T_{\varepsilon} b\right| \leq\left|\sum_{i} \sum_{j: 2^{j-2}>\varepsilon} B_{i} * K_{j}\right|+\sum_{i} \sum_{2^{j} \approx \varepsilon}\left|B_{i}\right| *\left|K_{j}\right| .
$$

There are no more than three $j$ terms appearing in the second sum, which is therefore bounded in $L^{1}$ norm by $C\|\Omega\|_{1}\|b\|_{1}$.

The first sum may be split into two parts, $T_{1, \varepsilon} b$ and $T_{2, \varepsilon} b$ where

$$
T_{1, \varepsilon} b=\sum_{i} \sum_{\substack{j=i+2 \\ 2^{j-2}>\varepsilon}}^{\infty} B_{i} * K_{j} .
$$

We need only prove (1) for $T_{1, \varepsilon} b$, because supp $T_{2, \varepsilon} b \subseteq E$. To see this, observe that for $j \leq i+1$ and $\left|Q_{n}\right|=2^{2 i}$, we have $\operatorname{supp}\left(K_{j} * b_{n}\right) \subseteq 5 Q_{n}$. Absolute convergence enables us to rearrange the summation defining $T_{1, \varepsilon} b$, so that

$$
T_{1, \varepsilon} b=\sum_{s \geq 2} \sum_{j: 2^{j-2}>\varepsilon} B_{j-s} * K_{j}
$$

By the inequalities of Minkowski and then Tchebychev, it suffices to prove

$$
\left\|\sum_{j: 2^{j-2}>\varepsilon} B_{j-s} * K_{j}\right\|_{L^{r}} \leq C 2^{-\gamma s} s^{\beta} \alpha^{(r-1) / r}\|b\|_{1}^{1 / r}
$$

for some $\gamma, \beta>0$, where $1 / r=(1+1 / q) / 2$. As in [C], this follows by interpolation from the following.

LEMMA 1. For some $\eta>0$,

$$
\text { PROOF OF LEMMA } 1 .
$$

$$
\left\|\sum_{j} B_{j-s} * K_{j}\right\|_{2}^{2} \leq C \alpha 2^{-\eta s} s\|\Omega\|_{\infty}^{2}\|b\|_{1} .
$$

$$
\begin{aligned}
& \left\|\sum_{j: 2^{j-2}>\varepsilon} B_{j-s} * K_{j}\right\|_{2}^{2}=\sum_{j}\left\|B_{j-s} * K_{j}\right\|_{2}^{2} \\
& \quad+2 \sum_{j}\left\langle B_{j-1-s} * K_{j-1}, B_{j-s} * K_{j}\right\rangle+2 \sum_{j}\left\langle B_{j-2-s} * K_{j-2}, B_{j-s} * K_{j}\right\rangle \\
& \quad+2 \sum_{j} \sum_{l \leq j-3}\left\langle B_{l-s} * K_{l}, B_{j-s} * K_{j}\right\rangle .
\end{aligned}
$$


By Lemma 2.1 of $[\mathbf{C}]$ and dilation invariance, the first sum on the right is bounded by $C 2^{-\eta s} \alpha\|\Omega\|_{\infty}^{2} \sum_{j}\left\|B_{j-s}\right\|_{1}$. The next two sums can be handled in the same fashion after applying Schwarz's inequality. To estimate the last sum define $\tilde{K}_{j}(x)=$ $K_{j}(-x)$ and note that

$$
\begin{aligned}
& \left|\sum_{j: 2^{j-2}>\varepsilon} \sum_{\substack{l: 2^{l-2}>\varepsilon \\
l \leq j-3}}\left\langle B_{l-s} * K_{l}, B_{j-s} * K_{j}\right\rangle\right| \\
& =\left|\sum_{j}\left\langle\left(\sum_{l} B_{l-s} * K_{l} * \tilde{K}_{j}\right), B_{j-s}\right\rangle\right| \\
& \leq \sum_{j=-\infty}^{\infty}\left\|B_{j-s}\right\|_{1}\left\|\sum_{l \leq j-3} B_{l-s} * K_{l} * \tilde{K}_{j}\right\|_{\infty} .
\end{aligned}
$$

To prove the lemma, it is now enough to show that

$$
\left\|\sum_{\substack{l \leq j-3 \\ 2^{l-2}>\varepsilon}} B_{l-s} * K_{l} * \tilde{K}_{j}\right\|_{\infty} \leq C \alpha 2^{-s} s\|\Omega\|_{\infty}^{2}
$$

Fix $x$ and $j$. Then there is an annulus $A_{j}(x)$ with $\left|A_{j}(x)\right|=C 2^{2 j}$ and $\operatorname{supp} K_{l} *$ $\tilde{K}_{j}(x-\cdot) \subseteq A_{j}(x)$, for all $l \leq j-3$. Thus, if $B_{l-s}=\sum_{n} b_{n}^{(l-s)}$ where $\operatorname{supp} b_{n}^{(l-s)} \subseteq$ $Q_{n}^{(l-s)}$ and $\left|Q_{n}^{(l-s)}\right|=2^{2(l-s)}$, then

$$
\left|\sum_{\substack{l \leq j-3 \\ 2^{l-2}>\varepsilon}} B_{l-s} * K_{l} * \tilde{K}_{j}(x)\right| \leq \sum_{l} \sum_{n}\left|\int b_{n}^{(l-s)}(y) K_{l} * \tilde{K}_{j}(x-y) d y\right|
$$

with the sum running over those $l$ and $n$ for which

$$
Q_{n}^{(l-s)} \cap A_{j}(x) \neq \varnothing \text {. }
$$

Note that since $l \leq j-3$ we have $\sum_{l} \sum_{n}\left|Q_{n}^{(l-s)}\right| \leq C 2^{2 j}$.

To prove (2), we will use

LEMMA 2. Fix s. Then for $l \leq j-3$, there is a decomposition $K_{l} * \tilde{K}_{j}=k_{1}^{l, j}+k_{2}^{l, j}$ such that

(i) $\left\|k_{1}^{l, j}\right\|_{\infty} \leq C\|\Omega\|_{\infty}^{2} 2^{-2 j} 2^{-s}$,

(ii) $\left\|\nabla k_{2}^{l, j}\right\|_{\infty} \leq C\|\Omega\|_{\infty}^{2} 2^{-2 j} 2^{-l} s$,

where $C$ is an absolute constant. Assume for now that Lemma 2 holds, so

$$
\begin{aligned}
& \sum_{l} \sum_{n} \int\left|b_{n}^{(l-s)}(y) k_{1}^{l, j}(x-y)\right| d y \\
& \quad \leq C\|\Omega\|_{\infty}^{2} 2^{-s} 2^{-2 j} \alpha \sum_{l} \sum_{n}\left|Q_{n}^{(l-s)}\right| \\
& \quad \leq C\|\Omega\|_{\infty}^{2} 2^{-s} \alpha .
\end{aligned}
$$


Also, with $y^{\prime}=$ center of $Q_{n}(l-s)$, since $b_{n}$ has mean value zero,

$$
\begin{aligned}
& \sum_{l} \sum_{n}\left|\int b_{n}^{(l-s)}(y) k_{2}^{l, j}(x-y) d y\right| \\
& \quad \leq \sum_{l} \sum_{n} \int\left|b_{n}^{(l-s)}(y)\right|\left|y-y^{\prime}\right|\left\|\nabla k_{2}^{l, j}\right\|_{\infty} d y \\
& \quad \leq C\|\Omega\|_{\infty}^{2} s 2^{-2 j} \sum_{l} \sum_{n} 2^{-l} 2^{l-s}\left\|b_{n}^{l-s}\right\|_{1} \\
& \quad \leq C\|\Omega\|_{\infty}^{2} 2^{-s} s \alpha .
\end{aligned}
$$

Modulo Lemma 2, this concludes the proof of (2), and hence of Lemma 1 and of the Theorem.

ProOF OF LEMMA 2. Since $K_{l} * \tilde{K}_{j}(x)=2^{-2 j} K_{l-j} * \tilde{K}_{0}\left(2^{-j} x\right)$, it is enough to prove that for $m \geq 3$ and $\delta>0$ given, there is a decomposition $k_{m, 1}+k_{m, 2}=$ $K_{-m} * \widetilde{K}_{0}$ such that

(i) $\left\|k_{m, 1}\right\|_{\infty} \leq C\|\Omega\|_{\infty}^{2} \delta$,

(ii) $\left\|\nabla k_{m, 2}\right\|_{\infty} \leq C\|\Omega\|_{\infty}^{2} 2^{m} \log \left(\delta^{-1}\right)$.

We will then take $m=j-l$ and $\delta=2^{-s}$.

Set $\psi(t)=t^{-1} \varphi(t)$ so that $K_{0}(x)=\Omega(x) \varphi(|x|)|x|^{-2}=\Omega(x) \psi(|x|)|x|^{-1}$ and $K_{-m}(x)=2^{2 m} K_{0}\left(2^{m} x\right)=2^{m} \Omega(x) \psi\left(2^{m}|x|\right)|x|^{-1}$.

As mentioned in the introduction, we have the representation

$$
K_{-m} * \tilde{K}_{0}(x)=\iint_{S^{1} \times S^{1}} u_{\theta}^{m} * \tilde{\mu}_{\omega}(x) d \theta d \omega
$$

where $\mu_{\theta}^{m}$ and $\tilde{\mu}_{\omega}$ are singular measures defined by

$$
\begin{aligned}
\left\langle\mu_{\theta}^{m}, h\right\rangle & \equiv \Omega(\theta) \int_{0}^{\infty} h(t \theta) 2^{m} \psi\left(2^{m} t\right) d t, \\
\left\langle\tilde{\mu}_{\omega}, h\right\rangle & \equiv \Omega(\omega) \int_{0}^{\infty} h(-s \omega) \psi(s) d s .
\end{aligned}
$$

The reader may verify (3) by using polar coordinates. Furthermore, by definition

$$
\left\langle\mu_{\theta}^{m} * \tilde{\mu}_{\omega}, h\right\rangle=2^{m} \Omega(\theta) \Omega(\omega) \int_{0}^{\infty} \int_{0}^{\infty} h(t \theta-s \omega) \psi\left(2^{m} t\right) \psi(s) d t d s .
$$

By making the change of variable $x=t \theta-s \omega$ in the last equation we have

$$
\left\langle\mu_{\theta}^{m} * \tilde{\mu}_{\omega}, h\right\rangle=\frac{2^{m} \Omega(\theta) \Omega(\omega)}{\Delta} \iint h\left(x_{1}, x_{2}\right) \psi\left(\frac{2^{m} \omega^{*} \cdot x}{\Delta}\right) \psi\left(\frac{\theta^{*} \cdot x}{\Delta}\right) d x_{1} d x_{2}
$$

with $\omega^{*}=\left(-\omega_{2}, \omega_{1}\right), \theta^{*}=\left(-\theta_{2}, \theta_{1}\right)$ and

$$
\Delta \equiv\left\|\begin{array}{ll}
\theta_{1} & -\omega_{1} \\
\theta_{2} & -\omega_{2}
\end{array}\right\|=\left|\omega^{*} \cdot \theta\right|
$$

Thus,

Note that

$$
\mu_{\theta}^{m} * \tilde{u}_{\omega}(x)=2^{m} \Omega(\theta) \Omega(\omega) \Delta^{-1} \psi\left(\frac{2^{m} \omega^{*} \cdot x}{\Delta}\right) \psi\left(\frac{\theta^{*} \cdot x}{\Delta}\right) .
$$

$$
\left|\nabla_{x} \mu_{\theta}^{m} * \tilde{\mu}_{\omega}(x)\right| \leq\left(C 2^{2 m} / \Delta^{2}\right)\|\Omega\|_{\infty}^{2} .
$$


Define $\|\omega-\theta\| \equiv \min \left(|\omega-\theta|_{S^{1}},|\omega+\theta|_{S^{1}}\right)$, where $|(\cdot)|_{S^{1}}$ denotes arc length measured along the circle. We have

$$
\Delta \equiv\left|\omega^{*} \cdot \theta\right| \approx\|\omega-\theta\| .
$$

Also, because $|x| \approx C>0$ for $x \in \operatorname{supp} K_{-m} * \tilde{K}_{0}(m \geq 3)$, and since supp $\psi \subseteq$ $\left(\frac{1}{4}, 1\right), \mu_{\theta}^{m} * \tilde{u}_{\omega}(x)$ is nonvanishing only if

$$
2^{m}\left\|\omega-x^{\prime}\right\| \approx \Delta \approx\left\|\theta-x^{\prime}\right\|
$$

where $x^{\prime}=x /|x|$.

Now set $K_{-m} * \tilde{K}_{0}=k_{m, 1}+k_{m, 2}$ with

$$
k_{m, 1} \equiv 2^{m} \iint_{\|\theta-\omega\| \leq \delta} \frac{\Omega(\theta) \Omega(\omega)}{\Delta} \psi\left(\frac{2^{m} \omega^{*} \cdot x}{\Delta}\right) \psi\left(\frac{\theta^{*} \cdot x}{\Delta}\right) d \theta d \omega .
$$

Then $k_{m, 1}$ and $k_{m, 2}$ satisfy the desired estimates.

\section{BIBLIOGRAPHY}

[C-Z] A. P. Calderon and A. Zygmund, On singular integrals, Amer. J. Math. 78 (1956), 289-309.

[C] M. Christ, Weak-type $(1,1)$ bounds for rough operators, preprint.

School of Mathematics, University of Minnesota, Minneapolis, Minnesota 55455

Current address (effective July 1, 1988): Department of Mathematics and Statistics, McMaster University, Hamilton, Ontario, Canada L8S, 4K1 